\title{
Perfil funcional de adultos com deficiência visual adquirida*
}

\section{Functional profile of adults with acquired visual disabilities}

\author{
Marina Menezes Born ${ }^{1}$, Karla da Silva Pereira ${ }^{1}$, \\ Gabriela Cordeiro Corrêa do Nascimento ${ }^{2}$
}

http://dx.doi.org/10.11606/issn.2238-6149.v26i2p244-249

Born MM, Pereira KS, Nascimento GCC. Perfil funcional de adultos com deficiência visual adquirida. Rev Ter Ocup Univ São Paulo. 2015 maio-ago.;26(2):244-9.

RESUMO: A perda da visão pode ocorrer em qualquer fase da vida, acarretando impactos referentes ao enfrentamento da nova condição, à identidade do sujeito e ao desempenho de suas atividades cotidianas. Frente a isso, buscou-se verificar se é possível traçar o perfil funcional de adultos com deficiência visual adquirida que frequentam uma instituição de habilitação e reabilitação de pessoas com deficiência visual do município de Curitiba-PR, por meio de pesquisa documental, quantitativa, de caráter exploratóriodescritivo. Para coleta e análise dos dados foi utilizado um roteiro baseado nos conceitos da Classificação Internacional de Funcionalidade, Incapacidade e Saúde (CIF). Foram analisados 27 registros, sendo que $33,3 \%(f=9)$ apontaram restrições no cuidado pessoal; $88,8 \%(f=24)$, na vida doméstica; $7,4 \%(f=2)$ no trabalho; $33,3 \%$ no lazer $(f=9)$; e $81,5 \%(f=22)$ na mobilidade. Foi possível obter indícios do perfil funcional desses sujeitos, no entanto, para um perfil mais detalhado, os dados documentais mostraram-se insuficientes. Os resultados encontrados permitiram uma reflexão sobre o tema, propondo novos estudos nesta área.

DESCRITORES: Baixa visão; Cegueira; Classificação internacional de funcionalidade, Incapacidade e saúde; Atividades cotidianas.
Born MM, Pereira KS, Nascimento GCC. Functional profile of adults with acquired visual disabilities. Rev Ter Ocup Univ São Paulo. 2015 May-Aug.;26(2):244-9.

\begin{abstract}
Vision loss can occur at any stage in life, causing impacts on facing the new condition, the subject's identity, and the performance of their daily activities. Therefore, this study intended to investigate the possibility of tracing the functional profile of adults with acquired visual disability who attend a habilitation and rehabilitation institution for visually impaired people in the city of Curitiba-PR, through quantitative, desk research of exploratory and descriptive nature. For the collection and analysis of data, a script based on the concepts of the International Classification of Functioning, Disability and Health (ICF) was used. Twenty seven records were analyzed, of which $33.3 \%(f=9)$ reported restrictions in personal care, $88.8 \%(f=24)$ in the household life, $7.4 \%(f=2)$ at work, $33.3 \%$ $(f=9)$ at leisure, and $81.5 \%(f=22)$ in mobility. It was possible to obtain evidence of the functional profile of these subjects; however, for a more detailed profile, the documentary data proved insufficient. The results allowed contemplation on the theme, proposing new studies in this area.
\end{abstract}

KEYWORDS: Vision, low; Blindness; International classification of functioning, Disability and health; Activities of daily living.

\footnotetext{
* Recorte do projeto de pesquisa intitulado "Perfil ocupacional de adultos com deficiência visual adquirida", do Departamento de Terapia Ocupacional da Universidade Federal do Paraná (UFPR), aprovado pelo Comitê de Ética do Setor de Ciências da Saúde sob o parecer de n ${ }^{\circ}$ 448.273, CAAE: 17485913.9.0000.0102. Parte dos resultados foi apresentada no XIII Congresso Brasileiro de Terapia Ocupacional e Políticas Públicas: diretrizes, compromissos e ações, Florianópolis-SC, 2013, sob forma de pôster eletrônico.

1. Terapeuta ocupacional graduada pela Universidade Federal do Paraná.

2. Professora do Departamento de Terapia Ocupacional da Universidade Federal do Paraná (UFPR).

Endereço para correspondência: Universidade Federal do Paraná. Campus Botânico, Bloco Didático II, Departamento de Terapia Ocupacional. Av. Lothário Meissner, 632. Bairro Jardim Botânico. Curitiba, PR. CEP: 80210-170. E-mail: gabriela.correa@ufpr.br.
} 


\section{INTRODUÇÃO}

A deficiência visual refere-se à incapacidade para enxergar, em maior ou menor grau, caracterizando-se como cegueira ou baixa visão. Pode ocorrer por fatores congênitos e ou hereditários, ou ainda ser adquirida em qualquer fase da vida devido a doenças oculares ou traumas que acarretem na perda brusca ou progressiva da visão ${ }^{1-4}$.

A deficiência visual adquirida implica a ruptura do automatismo geralmente envolvido no desempenho de atividades rotineiras. Além da perda da visão, a pessoa sofre com outras perdas que implicam restrições ao desenvolvimento, à mobilidade, às atividades profissionais, à execução das atividades cotidianas - como alimentação e vestuário, limpeza da casa -, entre outras. Assim, a pessoa depara com situações que a levam a uma condição de dependência e perda de autonomia para desempenhar tarefas que até então realizava independentemente $e^{3-7}$.

O sujeito é caracterizado e reconhecido pelas suas produções e pelo seu papel profissional, considerando a supervalorização dada a esses papéis na sociedade atual. Por meio do trabalho a pessoa regula e organiza sua vida, transforma a si mesmo e se engaja socialmente ${ }^{8-10}$. Portanto, quando o sujeito é afastado dessa atividade, sua identidade e papeis sociais são descontruídos ${ }^{8}$.

Em uma sociedade na qual a produção tem principal destaque $^{8}$, o lazer se torna um elemento importante da vida das pessoas e está diretamente relacionado com a qualidade de $\operatorname{vida}^{5,11,12}$. A participação em atividades de lazer pode ser limitada pela existência de barreiras para o desempenho, dentre elas: o contexto (arquitetônicas ou atitudinais), a demanda funcional exigida e/ou a presença de déficits em habilidades ${ }^{13,14}$.

Destaca-se que as restrições vivenciadas nas atividades (cuidado pessoal, vida doméstica, trabalho e lazer), pela pessoa que adquire a deficiência visual podem ser em decorrência da limitação na mobilidade. Perder ou ter a mobilidade prejudicada acarreta mudanças profundas, não só em relação à locomoção, mas também em relação à vida social ${ }^{15,16}$.

A Classificação Internacional de Incapacidade, Funcionalidade e Saúde (CIF) "permite descrever situações relacionadas com a funcionalidade do ser humano e as suas restrições e serve como enquadramento para organizar esta informação" (p.11) ${ }^{17}$. Assim, favorece a identificação das condições estruturais, ambientais e das características pessoais que interferem na funcionalidade do sujeito, podendo caracterizar-se como barreiras ou facilitadores do desempenho. A identificação desses fatores pode direcionar as intervenções, buscando otimizar os níveis de participação da pessoa com deficiência ${ }^{17}$.

A CIF fornece uma estrutura conceitual referente às funções e estruturas do corpo, atividades e participação e fatores ambientais, buscando estimular - dentre outras aplicações - a melhoria da participação do sujeito, minimizando barreiras e potencializando facilitadores ${ }^{17}$. Neste sentido, o presente estudo utilizou-se dessa estrutura para organizar o roteiro de coleta de dados, o registro das informações e a descrição e análise dos resultados.

A pesquisa objetivou identificar se é possível traçar o perfil funcional de pessoas com deficiência visual adquirida que frequentam uma instituição especializada voltada à habilitação e reabilitação de pessoas com deficiência visual do município de Curitiba (Paraná), por meio de análise documental. Para traçar o perfil funcional foi dado enfoque às atividades de cuidado pessoal, vida doméstica, trabalho e lazer. A escolha dos objetivos decorreu de demandas acadêmicas e institucionais, visando ampliar o conhecimento na área da deficiência visual, conhecer a população atendida e otimizar o direcionamento de serviços ofertados pela instituição parceira.

\section{PROCEDIMENTOS METODOLÓGICOS}

O estudo caracterizou-se como uma pesquisa documental, de caráter exploratório-descritivo, utilizando fontes primárias de informação, ou seja, documentos que ainda não haviam recebido tratamento analítico e/ou científico $^{18}$. A pesquisa exploratória constitui-se como o início de um processo de investigação, fornecendo maiores informações sobre o tema pesquisado e possibilitando a formulação de hipóteses futuras. A pesquisa descritiva busca descrever as características de determinada população ou fenômeno ${ }^{19}$.

Foram incluídos na pesquisa os registros de adultos, a partir de 18 anos, com deficiência visual adquirida, de ambos os sexos, que frequentam uma instituição de atendimento especializado. Essa é voltada à habilitação e reabilitação de pessoas com deficiência visual, oferecendo serviços como: orientação e mobilidade, Atividades de Vida Autônoma e Social (AVAS), Braille, Sorobã, informática, psicologia e assistência social. Outros serviços, como musicoterapia e Terapia Ocupacional, são ofertados por meio de parcerias externas.

O acesso ao acervo documental foi autorizado pela instituição e pelos sujeitos por meio da assinatura do Termo de Compromisso Livre e Esclarecido (TCLE), sendo que a amostra do estudo constituiu-se dos registros de 27 sujeitos. 
Dentre o acervo documental, foram encontrados no prontuário: ficha de identificação, contendo dados pessoais, socioeconômicos, condição de saúde e tipo de deficiência; laudos médicos sobre as deficiências; registros e relatórios quanto à participação dos sujeitos nas atividades ofertadas pela instituição; e instrumentos de avaliação. Esses dados provinham de diferentes profissionais: médicos, professores, pedagogos, terapeuta ocupacional e musicoterapeutas. Vale considerar que não existia uma sistematização das informações; assim, nem todos os documentos estavam disponíveis em todos os prontuários.

Como instrumento de coleta de dados foi utilizado um roteiro elaborado com base nos conceitos da CIF, visando guiar a pesquisa e organizar as informações coletadas. Nele, constavam quatro itens principais: a) fatores pessoais: idade, genero, condições de saúde, forma e tempo de acometimento da deficiência visual; b) funções e estruturas do corpo: deficiência (tipo de deficiência visual, etiologia, forma de acometimento); c) atividade e participação: limitações na atividade e restrições na participação; d) fatores ambientais: facilitadores e barreiras encontrados no desempenho. Ressalta-se que optou-se por utilizar a estrutura conceitual da CIF, pois essa permite organizar a informação de maneira útil, integrada e facilmente acessível ${ }^{17}$. Ainda, considerando o acervo documental disponível e a realidade institucional, não seria possível classificar o perfil funcional dos indivíduos utilizando a codificação da CIF.

Osdadosobtidosforamanalisadosquantitativamente com base no método de estatística descritiva simples, que consiste na síntese e descrição dos resultados ${ }^{19}$. Para tanto foi utilizado o programa Microsoft Office Excel 2010, resultando em dados de frequência relativa e absoluta. Os dados obtidos foram tabulados, utilizando-se códigos de identificação específicos para cada sujeito, com o intuito de manter o sigilo da identidade dos participantes.

\section{RESULTADOS}

De acordo com o método de análise utilizado, os resultados obtidos serão apresentados conforme estruturação utilizada no instrumento de coleta de dados - fatores pessoais e funções e estruturas do corpo, atividade e participação e fatores ambientais -, descrito nos procedimentos metodológicos e considerando as frequências relativa e absoluta $(\% / f)$.

Ao todo foram analisados os registros de 27 adultos com deficiência visual adquirida, com média de idade de 46,1 anos, variando entre 18 e 70 anos. A caracterização dos sujeitos é apresentada na Tabela 1 .
Tabela 1 - Características pessoais da população, segundo gênero, idade e deficiência visual $(\mathrm{n}=27)$

\begin{tabular}{|c|c|c|}
\hline & $\%$ & $f$ \\
\hline \multicolumn{3}{|l|}{ Sexo } \\
\hline Masculino & 48,1 & 13 \\
\hline Feminino & 51,9 & 14 \\
\hline \multicolumn{3}{|l|}{ Idade } \\
\hline $18-30$ & 22,2 & 6 \\
\hline $31-40$ & 7,4 & 2 \\
\hline $41-50$ & 25,9 & 7 \\
\hline $51-60$ & 25,9 & 7 \\
\hline Mais que 60 & 18,5 & 5 \\
\hline \multicolumn{3}{|c|}{ Deficiência visual } \\
\hline Cegueira & 81,5 & 22 \\
\hline Baixa visão & 18,5 & 5 \\
\hline
\end{tabular}

Entre as causas da deficiência visual, prevaleceu a retinopatia diabética, o que está ilustrado na Tabela 2 .

Tabela 2 - Etiologia da deficiência visual $(n=27)$

\begin{tabular}{l|c|c}
\hline & $\mathbf{\%}$ & $\boldsymbol{f}$ \\
\hline Retinopatia diabética & 18,5 & 5 \\
\hline Glaucoma & 14,8 & 4 \\
\hline Outros & 33,3 & 9 \\
\hline Não especificada & 33,3 & 9 \\
\hline
\end{tabular}

As restrições no cuidado pessoal em decorrência da deficiência visual foram encontradas no registro de 33,3\% $(f=9)$ dos sujeitos, conforme especificadas na Tabela 3. No que se refere à participação nas atividades de vida doméstica, apareceram restrições em 88,8\% $(f=24)$ dos registros, como detalhado na Tabela 4. Em relação a esses domínios, identificou-se que todos os sujeitos da amostra frequentavam aulas de AVAS, o que foi considerado um facilitador para o desempenho.

Tabela 3 - Restrições no cuidado pessoal $(n=9)$

\begin{tabular}{l|c|c}
\hline & $\mathbf{\%}$ & $\boldsymbol{f}$ \\
\hline Lavar-se & 22,2 & 2 \\
\hline Vestir-se & 11,1 & 1 \\
\hline Comer & 33,3 & 3 \\
\hline Cuidar da própria saúde & 11,1 & 1 \\
\hline Outras & 22,2 & 2 \\
\hline
\end{tabular}

$\mathrm{Na}$ atividade de trabalho, apenas 7,4\% $(f=2)$ dos registros trouxeram informações sobre essa área, sendo que os dois se referiam à vontade do sujeito de exercer trabalho voluntário. Quanto ao lazer, a restrição foi encontrada nos 
registros de $33,3 \%(f=9)$ da amostra total da pesquisa, conforme ilustrado na Tabela 5. Em relação às atividades de trabalho e lazer, não foram identificados registros de fatores ambientais que atuassem como facilitadores ou barreiras para o desempenho.

Tabela 4 - Restrições na vida doméstica $(n=24)$

\begin{tabular}{l|c|c}
\hline & $\mathbf{\%}$ & $\boldsymbol{f}$ \\
\hline Comprar & 50 & 12 \\
\hline Preparação de refeições & 8,3 & 2 \\
\hline Lavar roupas & 12,5 & 3 \\
\hline Limpar a casa & 12,5 & 3 \\
\hline Consertar roupas & 12,5 & 3 \\
\hline Outras & 4,2 & 1 \\
\hline
\end{tabular}

Tabela 5 - Restrições no lazer $(\mathrm{n}=9)$

\begin{tabular}{l|c|c}
\hline & $\mathbf{\%}$ & $\boldsymbol{f}$ \\
\hline $\begin{array}{l}\text { Arte e cultura (museu, cinema, } \\
\text { teatro e/ou parques) }\end{array}$ & 66,7 & 6 \\
\hline $\begin{array}{l}\text { Outros (viajar, cantar, dançar, } \\
\text { usar computador/internet) }\end{array}$ & 66,7 & 6 \\
\hline Artesanato (tricô, crochê, tear) & 33,3 & 3 \\
\hline $\begin{array}{l}\text { Atividades esportivas (fazer ginastica, } \\
\text { ir a academia, jogar vôlei e caçador) }\end{array}$ & 33,3 & 3 \\
\hline
\end{tabular}

Além das atividadesaque se pretendiadarênfaseneste estudo, dados sobre o domínio mobilidade destacaram-se nos registros estudados. Constatou-se que $81,5 \%(f=22)$ dos registros identificaram limitações e/ou restrições na mobilidade, sendo que, desses, $63,6 \%(f=14)$ referiam-se a limitações na mobilidade em geral e $36,4 \%(f=8)$, na mobilidade fora de casa. Daqueles registros que apontaram limitações e/ou restrições nesse domínio, em relação aos fatores ambientais: $13,6 \%(f=3)$ indicaram a família como barreira; $59,1 \%(f=13)$ a aula de OM como facilitador; e $27,3 \%(f=6)$ o uso da bengala como facilitador.

\section{DISCUSSÃO}

Os dados encontrados nos registros da população estudada acerca do predomínio da cegueira sobre a baixa visão diferem de outros estudos que mostram o predomínio da baixa visão $0^{15,16,20}$. No que diz respeito à etiologia da deficiência visual, destaca-se que os dados obtidos assemelham-se com os de outros estudos que apontam a retinopatia diabética e o glaucoma como as principais causas da deficiência visual adquirida ${ }^{1,16,21-23}$.

Os resultados apontaram que a amostra deste estudo apresentou restrições/limitações nos domínios de cuidados pessoais e vida doméstica. Não foram encontrados em outras pesquisas dados quantitativos referentes a esses aspectos, no entanto é descrito na literatura o impacto da deficiência visual adquirida no desempenho dessas atividades ${ }^{15,24,25}$.

Escócio et al. ${ }^{24}$ relatam um grande número de pessoas com deficiência visual que precisam de ajuda de outros para realizar atividades do dia a dia, verificando um alto índice de dependência, o que restringe sua qualidade de vida.

Os resultados desta pesquisa apontaram para o baixo número de informações referentes às atividades de trabalho, sendo estas amplamente discutidas por outros autores da área. Montilha ${ }^{20}$ identificou que o início ou retomada das atividades profissionais se destacaram entre as expectativas das pessoas com deficiência visual, correspondendo a $72 \%$ da amostra de seu estudo. Na pesquisa realizada por Monteiro e Montilha ${ }^{16}$, observou-se que, após adquirir a deficiência visual, apenas pequena porcentagem das pessoas continua trabalhando. Este dado se dá pela dificuldade da inclusão no mercado de trabalho brasileiro; pelos obstáculos que envolvem o engajamento profissional do deficiente visual; e também porque, após adquirir a deficiência, o afastamento do trabalho e a aposentadoria são frequentes.

No que tange à participação no lazer, novamente não foram encontrados dados quantitativos quanto ao desempenho de pessoas com deficiência visual adquirida nessas atividades. Contudo Arruda ${ }^{15}$ afirma que perder a visão implica outras perdas na vida do sujeito, destacando as restrições na participação de atividades de lazer. Muitas vezes essas atividades deixam de ser praticadas, como identificado nos registros da população estudada. Mesmo não tendo sido encontrados registros de facilitadores ou barreiras em relação à participação no lazer neste estudo, Cavalcanti ${ }^{13}$, Hanson e Jones ${ }^{14}$ apontam que diversos fatores interferem e restringem a participação nessas atividades, como a presença de barreiras físicas e atitudinais.

Os resultados encontrados neste estudo em relação às limitações e restrições na mobilidade assemelham-se com outros estudos realizados ${ }^{16,20}$. No trabalho de Monteiro e Montilha ${ }^{16}$, a maioria dos sujeitos colocou a mobilidade como um dos principais objetivos no processo de reabilitação. A orientação e mobilidade foram indicadas por $76 \%$ dos sujeitos como expectativa de resultados do processo de reabilitação no estudo de Montilha ${ }^{20}$.

\section{CONCLUSÃO}

Os resultados obtidos a respeito das atividades de cuidado pessoal, vida doméstica, trabalho e lazer, levantaram indícios das limitações em relação ao 
desempenho nessas áreas. No entanto, considerando a escassez de informações e a forma de registro encontrada no acervo documental, não foi possível traçar um perfil funcional mais detalhado, identificando a causa das restrições e/ou limitações encontradas e os fatores ambientais que atuam como barreiras e facilitadores de algumas das atividades abordadas. Não foram encontradas informações a respeito da funcionalidade desses sujeitos no contexto e da participação deles na comunidade.

Considerando os dados enconrados no acervo documental, a metodologia utilizada na pesquisa e o referencial teórico sobre o tema, levanta-se a hipótese de que é possível que as limitações no desempenho podem apenas não estar registradas no acervo documental, embora estejam presentes na vida do sujeito. Assim, manifesta-se a necessidade de novos estudos diretamente com os sujeitos, a fim de identificar as reais limitações nas áreas

\section{REFERÊNCIAS}

1. Ribeiro LB. Disfunção visual. In: Souza ACA. Terapia ocupacional: fundamentação \& prática. Rio de Janeiro: Guanabara Koogan; 2007.

2. World Health Organization. Vision 2020 - the right to sight. Action plan 2006-2011. Geneva; 2007. Available from: http://www.who.int/blindness/Vision2020 report.pdf.

3. Parnof D. Deficiência visual: a perda e a superação. Rev Inst Benjamin Constant (Rio de Janeiro). 2010;16(45):29-37.

4. Mancini MC, Braga MAF, Albuquerque KA, Ramos TMV Chagas PSC. Comparação do desempenho funcional de crianças com visão subnormal e crianças com desenvolvimento normal aos 2 e 6 anos de idade. Rev Ter Ocup Univer São Paulo. 2010;21(3):215-22. DOI: http:// dx.doi.org/10.11606/issn.2238-6149.v21i3p215-222

5. Souza ELV, Moura GN, Nascimento JC, Lima MA, Pagliuca LMF, Caetano JA. Diagnóstico de enfermagem na teoria do autocuidado em pessoas com deficiência visual. Rev Rene. 2012;13(3):542-51. Disponível em: http://www.revistarene. ufc.br/revista/index.php/revista/article/view/721/pdf.

6. Gil M, editor. Cadernos da TV escola: deficiência visual. Brasília: Ministério da Educação, Secretaria de Educação a Distância; 2000.

7. Arruda SMCP. Atividades da vida diária: intervenção no processo de formação da pessoa com deficiência visual. de desempenho e como os fatores ambientais interferem na participação.

Utilizar a estrutura conceitual da CIF para a pesquisa mostrou-se pertinente à medida que auxiliou na organização e registro dos dados e na análise e discussão dos resultados. É necessário ponderar que, considerando a metodologia adotada e a realidade e natureza institucional, não se pretendia classificar os sujeitos pela CIF. Neste sentido, cabe refletir sobre a importância da utilização de uma linha funcional desde o momento da triagem e registro das pessoas que frequentam esse tipo de instituição, conforme prevê a CIF, a fim de obter um registro mais completo sobre o desempenho e a participação dos sujeitos. Esse tipo de organização pode auxiliar a tomada de decisões dos profissionais e otimizar o direcionamento de serviços ofertados pela instituição de acordo com as necessidade individuais do sujeito.

In: Quevedo AAF, Oliveira JR, Mantoan MTE. Mobilidade e comunicação: desafios à tecnologia e inclusão social. Campinas: Edição do Autor; 1999. p.133-40.

8. Rodrigues M, Ayabe NH, Lunardelli MCF, Canêo LC. A preparação para a aposentadoria: o papel do psicólogo frente a essa questão. Rev Bras Orientac Prof. 2005;6(1):5362. Disponível em: http://pepsic.bvsalud.org/scielo. php?pid=S1679-33902005000100006\&script=sci_arttext.

9. Neres CC; Corrêa NM. O trabalho como categoria de análise na educação do deficiente visual. Cad Cedes, Campinas. 2008;28(75):149-70. Disponível em http://www.scielo.br/ pdf/ccedes/v28n75/v28n75a02.pdf.

10. Toldrá RC. Políticas afirmativas: opinião das pessoas com deficiência acerca da legislação de reserva de vagas no mercado de trabalho. Rev Ter Ocup Univ São Paulo. 2009; 20(2):110-7. DOI: http://dx.doi.org/10.11606/issn.22386149.v20i2p110-117

11. Queiroz MNG, SouzaLK. Atividades de lazer em jovens e adultos: um estudo descritivo. Licere (Belo Horizonte). 2009;12(3):1-21. Disponível em: https://seer.lcc.ufmg.br/ index.php/licere/article/view/573/462.

12. Davim RMB, Dantas SMM, Lima VM, Lima JFV. O lazer diário como fator de qualidade de vida: o que pensa um grupo da terceira idade. Ciên Cuidado 
Saúde (Maringá). 2003;2(1):19-24. DOI: http://dx.doi. org/10.4025/cienccuidsaude.v2i1.5563.

13. Cavalcanti A. Avaliação de recreação e do lazer. In: Souza ACA. Terapia ocupacional: fundamentação \& prática. Rio de Janeiro: Guanabara Koogan; 2007.

14. Hanson C, Jones D. Restauração da competência na atividade de lazer. In: Trombly CA, Radomski MV. Terapia ocupacional para disfunções físicas. 5a ed. São Paulo: Santos; 2005.

15. Arruda SMCP. Acessibilidade no cotidiano de pessoas com deficiência visual. Rev@mbienteeduc (São Paulo). 2008;1(2):113-21. Disponível em: http://arquivos. cruzeirodosuleducacional.edu.br/principal/old/revista educacao/pdf/volume_2/rev_n\%C2\%BA2_10_arruda.pdf.

16. Monteiro MMB, Montilha RC. Reabilitação grupal: expectativas e percepções de portadores de deficiência visual. Medicina (Ribeirão Preto). 2012;45(1):66-77. DOI: http:// dx.doi.org/10.11606/issn.2176-7262.v45i1p66-77.

17. Organização Mundial da Saúde. CIF - Classificação Internacional de Funcionalidade, Incapacidade e Saúde. Lisboa; 2004. p.11.

18. Sá-Silva JR, Almeida CD, Guindani JF. Pesquisa documental: pistas teóricas e metodológicas. Rev Bras Hist Ciên Sociais. 2009;1(1):1-15. Disponível em: http://www. unisc.br/portal/upload/com_arquivo/pesquisa_documental pistas_teoricas_e_metodologicas.pdf

19. Silva AA. Prática clínica baseada em evidências na área da saúde. São Paulo: Editora Santos; 2009.

Recebido para publicação: 22.12 .14

Aceito para publicação: 24.06.15
20. Montilha RCI, Temporini ER, Kara-Jose N, Nobre MIRS. Deficiência visual: características e expectativas da clientela de serviços de reabilitação. Rev Ciênc Méd (Campinas). 2010; 9(3):123-8. Disponível em: http://periodicos.puccampinas.edu.br/seer/index.php/cienciasmedicas/article/ view/1328/1302.

21. Portes LCP, Portes ALF. Causas de baixa visão e cegueira no adulto. In: Kara-José N, Rodrigues MLV. Saúde ocular e prevenção da cegueira - Tema oficial do XXXV Congresso Brasileiro de Oftalmologia, 2009. Rio de Janeiro: Cultura Médica; 2009.

22. Viquez MV. Retinopatia diabética. Rev Costarric Salud Pública (San José). 2011;20(2):71-4. Disponível em: http:// www.scielo.sa.cr/scielo.php?script $=$ sci_arttext\&pid $=$ S1409$14292011000200001 \& \operatorname{lng}=\mathrm{en} \& n r m=\mathrm{iso} \& \operatorname{lng}=\mathrm{es}$.

23. Aliseda D, Berastegui L. Retinopatia diabética. An Sist Sanit Navar. 2008;31(Supl 3):23-34. Disponível em: http:// scielo.isciii.es/pdf/asisna/v31s3/original3.pdf.

24. Escócio J, Caetando JA, Soares E. O perfil do deficiente visual no município de Sobral/CE. Rev Rene (Fortaleza). 2004;5(2):49-56. Disponível em: http://www.revistarene. ufc.br/revista/index.php/revista/article/view/923/pdf.

25. Early MB. Desempenho ocupacional. In: Pedretti LW, Early MB. Terapia ocupacional: capacidades práticas para as disfunções físicas. 4a ed. São Paulo: Roca; 2005. 